\title{
WEAK SOLUTIONS OF ABSTRACT DIFFERENTIAL EQUATIONS
}

\author{
By M. A. Malik
}

DEPARTMENT OF MATHEMATICS, UNIVERSITY OF MANITOBA, WINNIPEG, CANADA

Communicated by Einar Hille, February 3, 1967

1. Let $H$ be a Hilbert space. Consider in $H$ a closed linear operator $A$ with dense domain $D_{A}$ and its adjoint $A^{*}$ with domain $D_{A^{*}}$. The domains $D_{A}$ and $D_{A^{*}}$ are Hilbert spaces under their respective graph-scalar products. We denote by $D(R), D\left(R ; D_{A^{*}}\right), \mathrm{S}^{\prime}(R ; H)$, and $D^{\prime}(R ; H)$ the spaces of infinitely differentiable functions with compact support, infinitely differentiable $D_{A} *$-valued functions with compact support, $H$-valued temperate distributions, and $H$-valued distributions, respectively, on $R$ with their usual topologies. ${ }^{3}$ By $\mathfrak{H C}^{s}(R ; H), s$ real, we mean the space of all $u \in S^{\prime}(R ; H)$ whose Fourier transform $\hat{u}(t)$ is a function (H-valued) and

$$
\int_{R}\left(1+|t|^{2}\right)^{s}|\hat{u}(t)|^{2} d t<\infty .
$$

Let $\mathcal{F}_{\text {loc }}^{s}(R ; H)$ be the space of those $u \in D^{\prime}(R ; H)$ for which $\phi u \in \mathcal{F}^{8}(R ; H)$ for every $\phi \in \mathscr{D}(R)$.

We denote by $L_{\text {loc }}^{2}(R ; H)$ the space of $H$-valued functions $f$ such that the norm $|f(t)|$ is square integrable on every compact set of $R$. For nonnegative integers, we present another definition of $\mathcal{H C}_{\mathrm{loc}}^{-s}(R ; H)$ by

$$
\mathcal{H}_{\mathrm{loc}}^{-s}(R ; H)=\left\{\sum_{k=0}^{s} D^{(k)} T_{k} ; T_{k} \in L_{\mathrm{loc}}^{2}(R ; H)\right\}
$$

$D^{(k)}=\frac{d^{k}}{d t^{k}}$ is a weak differential operator of order $k$.

The equivalence of these two definitions of $\mathfrak{H C}_{\text {loc }}^{-s}(R ; H)$ for any nonnegative integer $s$ may be verified using the partitions of unity. ${ }^{2}$

2. We need the following definitions in view of imposing conditions on the operator $A$.

Definition: Let $\mathcal{F}$ be a family of parallel lines $\left\{\left(\operatorname{Im} \lambda=\zeta_{n}\right)^{\infty}{ }_{-\infty}: \zeta_{n} \rightarrow \infty\right.$ as $n \rightarrow \infty ; \zeta_{n} \rightarrow-\infty$ as $\left.n \rightarrow-\infty\right\}$ in the complex $\lambda$-plane. Let $r$ be a positive real number and $j, m$ be integers. We shall say that the resolvent $R\left(\lambda ; A^{*}\right)=(\lambda I-$ $\left.A^{*}\right)^{-1}$ is of $(j, r, m)$-growth on $\mathcal{F}$ if $R\left(\lambda ; A^{*}\right)$ exists for $\lambda$ (let $|\lambda|>1$ ) outside $j$ intervals of length $r$ on every line of $\mathcal{F}$ and for these $\lambda$

$$
\left|R\left(\lambda ; A^{*}\right)\right|<\text { const. }|\lambda|^{m} \text {. }
$$

For $m=0$ in the definition, see reference 1, p. 131, where S. Agmon and L. Nirenberg have defined $(j, r)$-bounded resolvent on $\mathcal{F}_{+}(\mathcal{F}$ being in the upper half plane).

The aim of this note is to announce a generalization of a result due to S. Zaidman ${ }^{4}$ in the following

TheOREm. If $R\left(\lambda ; A^{*}\right)$ is of $(j, r, m)$-growth on $\mathcal{F}$, then for every $f \in \mathcal{F}_{\mathrm{loc}}^{m-s}(R ; H)$ the abstract differential equation $\frac{1}{i} \frac{d u}{d t}-A u=f$ has at least one weak solution $u \in \mathcal{H}_{\mathrm{loc}}^{-s}$ (R;H), i.e., 


$$
\left\langle u, \frac{1}{i} \frac{d \phi}{d t}-A^{*} \phi\right\rangle=\langle f, \phi\rangle
$$

for all $\phi \in \mathbb{D}\left(R ; D_{A^{*}}\right) ; s$ and $s-m$ are nonnegative integers.

For $m=s=0$, we have the theorem of Zaidman.

${ }^{1}$ Agmon, S., and L. Nirenberg, "Properties of solutions of ordinary differential equations in Banach space," Comm. Pure Appl. Math., 16, 121-239 (1963).

2 Gel'fand, I. M., and G. E. Shilov, Generalized Functions (New York: Academic Press, 1964).

${ }^{3}$ Hörmander, L., Linear Partial Differential Operators (New York: Academic Press, 1963).

" Zaidman, S., "A global existence theorem for some differential equations in Hilbert spaces," these Proceedings, 51, 1019-1022 (1964). 